\title{
Ce que disent les mots. Nommer la ville lusitanienne
}

What Words Say. Naming the Lusitanian City

\section{Susana Marcos}

\section{(2) OpenEdition \\ 1 Journals}

Édition électronique

URL : https://journals.openedition.org/gaia/1441

DOI : 10.4000/gaia. 1441

ISSN : 2275-4776

Éditeur

UGA Éditions/Université Grenoble Alpes

\section{Édition imprimée}

ISBN : 978-2-37747-199-7

ISSN : 1287-3349

\section{Référence électronique}

Susana Marcos, "Ce que disent les mots. Nommer la ville lusitanienne », Gaia [En ligne], 22-23 | 2020, mis en ligne le 30 juin 2020, consulté le 09 décembre 2021. URL : http://journals.openedition.org/gaia/ 1441 ; DOI : https://doi.org/10.4000/gaia.1441

Ce document a été généré automatiquement le 9 décembre 2021.

Gaia. Revue interdisciplinaire sur la Grèce archaïque 


\title{
Ce que disent les mots. Nommer la ville lusitanienne
}

\author{
What Words Say. Naming the Lusitanian City
}

\author{
Susana Marcos
}

S'interroger sur « Nommer la ville ${ }^{1} »$, c'est questionner le vocabulaire mais c'est aussi, de façon plus implicite, réfléchir à ce qu'est la ville, c'est-à-dire comment elle peut être définie, voire comment elle se définit elle-même. Mais qu'entendons-nous en réalité à travers le concept de ville? Dans l'Antiquité romaine, cette notion ne correspond pas exactement à celle qui est la nôtre à l'heure actuelle. Trop souvent, nous avons tendance à transposer à l'Antiquité des concepts contemporains que les Romains ignoraient. Fustel de Coulanges ${ }^{2}$ l'écrivait déjà : la ville n'est pas la cité au sens où la communauté précède bien souvent l'écrin dans lequel elle s'établit et qu'incarne l'Vrbs dont le terme insiste sur l'aspect extérieur, sur la monumentalité et l'urbanisme. Ainsi la notion de ville revêt-elle de multiples aspects ${ }^{3}$. Chez les Romains, cette ville est matérialisée par la cité, la ciuitas, le symbole par excellence de l'empreinte de Rome, l'instrument de réorganisation de tout l'Empire. Constituée du noyau urbain autour duquel se réunit une communauté et du territoire qui en dépend, la pertica sur laquelle la caput ciuitatis exerce son autorité, elle forme une circonscription à l'intérieur de laquelle s'exerce le pouvoir judiciaire local et l'autorité de Rome ${ }^{4}$. En réalité, c'est par analogie que le même mot désigne cet ensemble spatial et administratif. À l'origine, la ciuitas est avant tout une communauté politique capable de se gouverner, la constitutio du populus, qui implique par extension la citoyennetés. La ville peut donc être assimilée aux personnes qui l'habitent, qui y vivent et qui la nomment. Ce n'est qu'ensuite qu'elle désigne un centre urbain inscrit dans un territoire, la ville au sens large. Comme l'écrit Patrick Le Roux ${ }^{6}$, les travaux menés sur les cités lusitaniennes ont jusqu'à ce jour essentiellement suivi trois axes: tout d'abord l'identification même des ciuitates, leur localisation ainsi que l'histoire urbaine de façon plus générale ${ }^{7}$; les dimensions politiques, juridiques et commerciales qui permettent d'envisager la cité comme centre de pouvoir ${ }^{8}$; et enfin les questions territoriales et administratives sur lesquelles nous reviendrons ultérieurement. Le cadre de notre étude ne nous permet pas de revenir sur 
le détail de cette riche historiographie pour laquelle nous nous permettrons de renvoyer aux ouvrages de référence. Autant dire que ces réflexions constituent une base essentielle pour l'analyse de notre sujet.

C'est précisément dans un contexte de restructuration administrative, signe de maîtrise du territoire, que naît la province de Lusitanie. Une fois achevées les guerres contre les Astures et les Cantabres, vraisemblablement entre 16 à 13 av. J.-C., Auguste aurait procédé à ce nouveau découpage ${ }^{9}$ d'un espace géographiquement périphérique de l'Occident romain, de ces terres jusque-là peuplées d'une multitude de populi qui ont chacun leur chef, leur langue, leur culture et leurs coutumes. La prouincia est donc un cadre nouveau qui restructure les communautés ${ }^{10}$ par la mise en place d'un maillage hiérarchisé de nouvelles unités politico-juridiques: c'est dans ce contexte de stabilisation des populations et de réorganisation administrative qu'apparait la ciuitas. L'exemple lusitanien met ainsi en lumière cette période clé qu'est l'insertion de ces populations dans les cadres romains, moment qui se traduit par un glissement sémantique dans la manière de "nommer la ville » qu'il est bon de questionner. C'est la raison pour laquelle notre réflexion s'attachera à l'analyse et à la comparaison de cette évolution chronologique lexicale à la lumière du tableau administratif de référence que dresse Pline l'Ancien de la province ${ }^{11}$. Notre propos n'est pas ici d'apporter une réponse définitive, ou encore moins de statuer sur l'intégration des communautés au monde romain. Par contre, l'étude sera l'occasion d'éclairer comment les mots sont révélateurs tout autant des usages quotidiens des populations locales que de l'évolution des conceptions politiques venues de Rome.

L'Histoire naturelle est sans aucun doute l'une des sources fondamentales pour la connaissance des cités de Lusitanie. Néanmoins, « dans la mesure où le langage de Pline se diversifie au fil du texte, la question se pose des nuances à introduire ou non entre les différents vocables utilisés, à savoir oppidum, ciuitas et populus ${ }^{12} »$ : le lexique rend bien compte de la complexité des organisations préexistantes auxquelles doivent s'adapter les Romains lors de la mise en place de la nouvelle province. La description de la Lusitanie débute au livre IV, 116. Là, Pline atteste l'existence de quatre gentes, c'est-àdire des groupes ethno-historiques, qui vivent sur ces terres : les Celtici et les Turduli au sud; à l'est, au-delà du Côa, dans les régions de Cáceres et Salamanca actuelles, se trouvent les Vettons, et enfin les Lusitaniens qui donnèrent leur nom à la prouincia. Malgré l'importance qui leur est accordée, ils occupent un espace assez limité, compris entre le Douro et le Tage. De la même manière, la fixation de la province, qui n'a pas d'existence propre auparavant et au sein de laquelle priment les relations bilatérales entre Rome et les communautés locales, émane d'une décision impériale, elle-même basée sur une vision géographique et une terminologie bien plus ancienne ${ }^{13}$. Devenus romains, ces peuples sont regroupés pour faciliter la gestion administrative, notamment la collecte $\mathrm{du}$ stipendium au travers $\mathrm{du}$ cadre qu'est la ciuitas. Paradoxalement, si nous reprenons les listes que dresse Pline l'Ancien, il est frappant de constater qu'il introduit le passage qui sert de base à toute étude des cités lusitaniennes en faisant référence non pas à la ciuitas, mais aux populi qui peuplent la Lusitanie: "Tota populorum XLV, in quibus coloniae sunt quinque, municipium ciuium Romanorum, Latii antiqui III, stipendiariae XXXVI ${ }^{14}$. » La Lusitanie est un ensemble culturel extrêmement morcelé dont le manque de cohésion et d'unité est pour les Romains synonyme de désorganisation. Depuis toujours, ces régions sont des terres de passage et de migrations ${ }^{15}$. 
Comme l'atteste l'étude archéologique récemment menée par Pedro Carvalho ${ }^{16}$, les populi de la Lusitanie septentrionale constituent tout un réseau de petits hameaux, visibles dans le paysage et aux bonnes conditions de défense, qui fonctionnent chacun de façon indépendante et contrôlent les voies de passages naturels. Ainsi, le terme de Lusitani est un ethnonyme que les Romains utilisaient sous la République pour désigner l'ennemi, mais qui regroupe une multitude de populi, dont nous ignorons les caractéristiques communes ${ }^{17}$. Faute de traces matérielles, ils sont tout aussi difficiles à identifier qu'à localiser. La description est d'autant plus complexe que Pline regroupe sous le nom de populus des statuts juridiques romains très divers comme cinq colonies, un municipe de droit romain, trois communautés de droit latin et trente-six cités stipendiaires. Peut-être cela pourrait-il s'expliquer par les sources plus anciennes qu'utilise l'auteur pour écrire, notamment la Formula Prouinciarum d'Agrippa? Dans ce cas, il serait compréhensible qu'à cette époque survive une organisation fondée sur la base indigène, le populus, en tant que communauté politique organisée, et non sur la cellule de base qu'est la ciuitas de type romain.

De façon tout aussi surprenante, à aucun moment il n'utilise le terme de ciuitas : il ne fait mention que d'oppidum ${ }^{18}$. Il en évoque certains par le nom du lieu au singulier comme Olisippo, Salacia, Ossonoba, Balsa, Myrtilis ${ }^{19}$, tandis que les oppida stipendiaria sont cités par le nom des habitants au pluriel : «Stipendiariorum quos nominare non pigeat, [...] Augustobrigenses, Aeminienses, Aranditani, Arabricenses, Balsenses, $[. ..]{ }^{20} . »$ La tradition urbaine plus ou moins ancienne de ces régions respectives explique probablement cette différence. En effet, dès l'âge de bronze, l'aire culturelle celtico-turdule connaît des structures d'habitat qui s'apparentent aux villes, aussi embryonnaire fussent-elles: Ossonoba, Balsa, Myrtilis, Salacia, Olisipo, fonctionnent comme des cités-États ${ }^{21}$. $\mathrm{Au}$ contraire, les terres septentrionales sont davantage celles de populi que nous avons évoqués, et que traduit bien l'emploi du pluriel de Pline. Pour ce dernier, l'oppidum est donc la ville, quel que soit son statut juridique ${ }^{22}$; pour autant, il ne peut être confondu d'un point de vue juridique avec la ciuitas. En qualifiant toutes les capitales de ciuitates, le terme se réfère à un élément de l'organisation territoriale, un centre politique et administratif qui contrôle d'autres noyaux de peuplement ${ }^{23}$. De ce point de vue, il est compréhensible que Pline l'Ancien comptabilise ainsi les cités : il utilise ce concept pour désigner des collectivités qui participent à ce processus. C'est la raison pour laquelle le terme disparaît progressivement et est remplacé par celui de ciuitas.

6 "L'histoire romaine débute sous le signe de l'obsession des confins. Délimiter, marquer son territoire: tel est l'enjeu de la fondation de la Ville - et de toute ville ${ }^{24}$.» Les termini augustales qui délimitent les territoires récemment conquis marquent une étape fondamentale de l'occupation romaine: celui du partage du territoire entre de multiples ciuitates ${ }^{25}$. La superficie totale de la pertica, c'est-à-dire le territoire dépendant de la cité, est établie à partir de la délimitation de son périmètre : Rome calcule ainsi la somme globale que la communauté doit lui payer, laissant à celle-ci la compétence de répartir individuellement le tribut ${ }^{26}$. À ce titre, le cas du territoire d'Augusta Emerita, la capitale de province, fait figure d'exemplum en raison de ses dimensions hors du commun $^{27}$. L'objectif est de protéger l'espace délimité et de le distinguer de celui de ses voisins de manière à éviter les disputes territoriales, définissant ainsi le territoire sur lequel les magistrats locaux exercent leur juridiction et prélèvent l'impôt ${ }^{28}$. Pour ce faire, la ligne de démarcation doit être correctement signalée, visible et compréhensible de tous. De nombreux éléments naturels comme la ligne de partage des 
eaux ou de crêtes, des pierres remarquables par leur taille ou leur forme ${ }^{29}$, ont permis de définir ces espaces. Malheureusement dans ces cas, il ne nous reste plus rien du tracé ; d'où l'importance des documents épigraphiques qui délimitent la pertica civique. À ce jour, nous pouvons dénombrer quinze exemplaires de termini augustales en Lusitanie (tableau 1$)^{30}$.

Tableau 1. - Les termini augustales de Lusitanie.

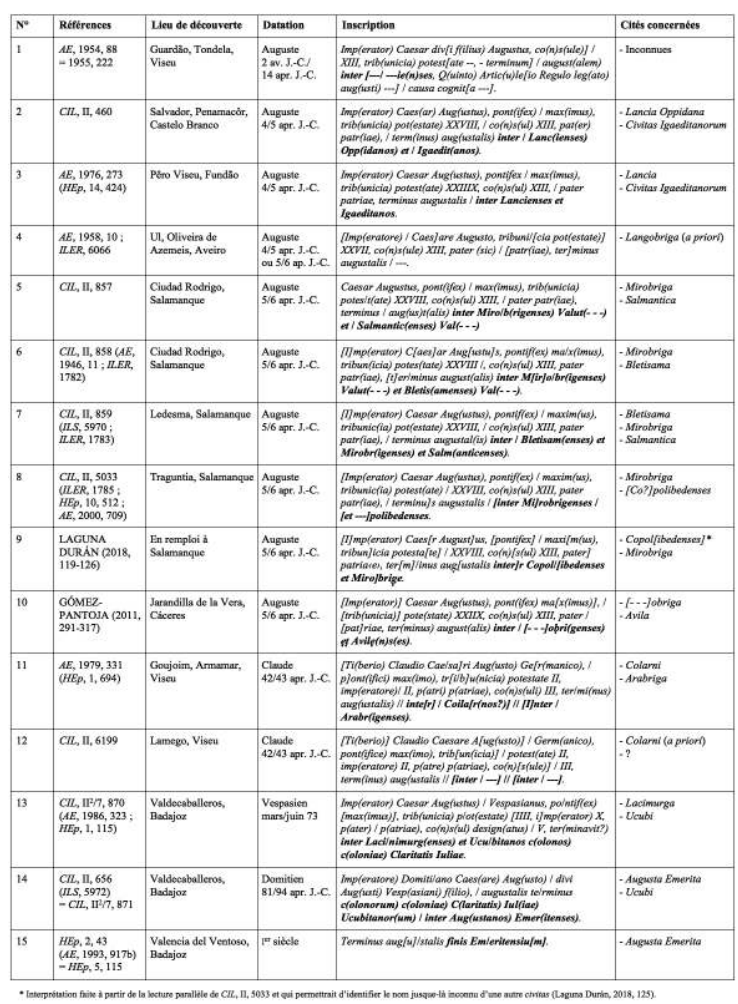

7 D'un point de vue strictement formel, la majorité des bornes conservées ont une forme rectangulaire ou de cippe qui n'est pas sans rappeler celle des autels ${ }^{31}$, aspect qui pourrait être mis en lien avec la sacralité des limites ${ }^{32}$ et qui assure le maintien de l'ordre public. Dans ce cas précisément, la nécessaire visibilité sur le territoire civique et le fait d'inscrire son nom revêtent une importance fondamentale que corrobore le caractère commémoratif du formulaire de ces monuments ${ }^{33}$ : c'est l'occasion pour la cité de s'affirmer visuellement. Nommer la ville, c'est donc dire son existence, la distinguer d'une autre. Concernant l'analyse du texte épigraphique, nous pouvons remarquer la constance du terme augustalis: autrement dit, la décision de l'organisation territoriale émane de l'autorité impériale, mettant ainsi fin à tout contentieux éventuel. Dans la pratique, c'est le pouvoir central romain qui valide la terminatio après avis des mensores ${ }^{34}$. Ce qui est particulièrement frappant dans ce corpus d'inscriptions, c'est qu'à cette époque haute, ni la catégorie juridique des cités en question, ni le terme ciuitas lui-même, ne sont jamais indiqués, rejoignant ici le texte plinien. La mention des communautés délimitées se fait par la seule utilisation d'un accusatif pluriel, comme l'exige l'emploi de la préposition inter ${ }^{35}$. Par conséquent, il est remarquable qu'à chaque fois, le terme utilisé renvoie systématiquement, non pas à une unité administrative, mais à des habitants, ce qui n'est pas sans rappeler l'héritage ethnique local que sont la multitude de petits populi auxquels nous faisions référence précédemment. En outre, la datation de ces opérations de bornage ainsi que 
l'homogénéité des formulaires mettent en avant l'œuvre augustéenne ${ }^{36}$ qui s'exerce sur un temps très court, à un moment clé de transition entre l'organisation préexistante et le nouveau modèle imposé par le pouvoir impérial de la ciuitas: trois termini datent de 4-5 apr. J.-C. ${ }^{37}$ et six autres de 5-6 apr. J.-C. ${ }^{38}$. Les autres textes sont d'époque claudienne $^{39}$ et flavienne ${ }^{40}$. Le fait que deux d'entre eux aient été retrouvés à Valdecaballeros indique que ce lieu stratégique a été le fruit de la convoitise des cités voisines : il est fort possible que la restructuration de l'espace civique ait pu entraîner certains contentieux, voire qu'il ait été nécessaire de la repréciser avec le temps. Le rôle de l'empereur est alors davantage centré sur la consolidation des territoires. Malgré les aléas des découvertes que nous ne pouvons ignorer, le contraste entre le nord et le sud de la province est néanmoins frappant (fig. 1 ${ }^{41}$. En effet une opération d'envergure entre 4 et 6 apr. J.-C. se centre sur une vaste région comprise entre le Douro et le Tage où ont été retrouvées neuf inscriptions. Cinq plus particulièrement proviennent de la région de Salamanque et concernent les mêmes cités : Mirobriga, Bletisama, Salmantica et les Copolibedenses (? $)^{42}$.

Fig. 1. - Répartition géographique des termini augustales.

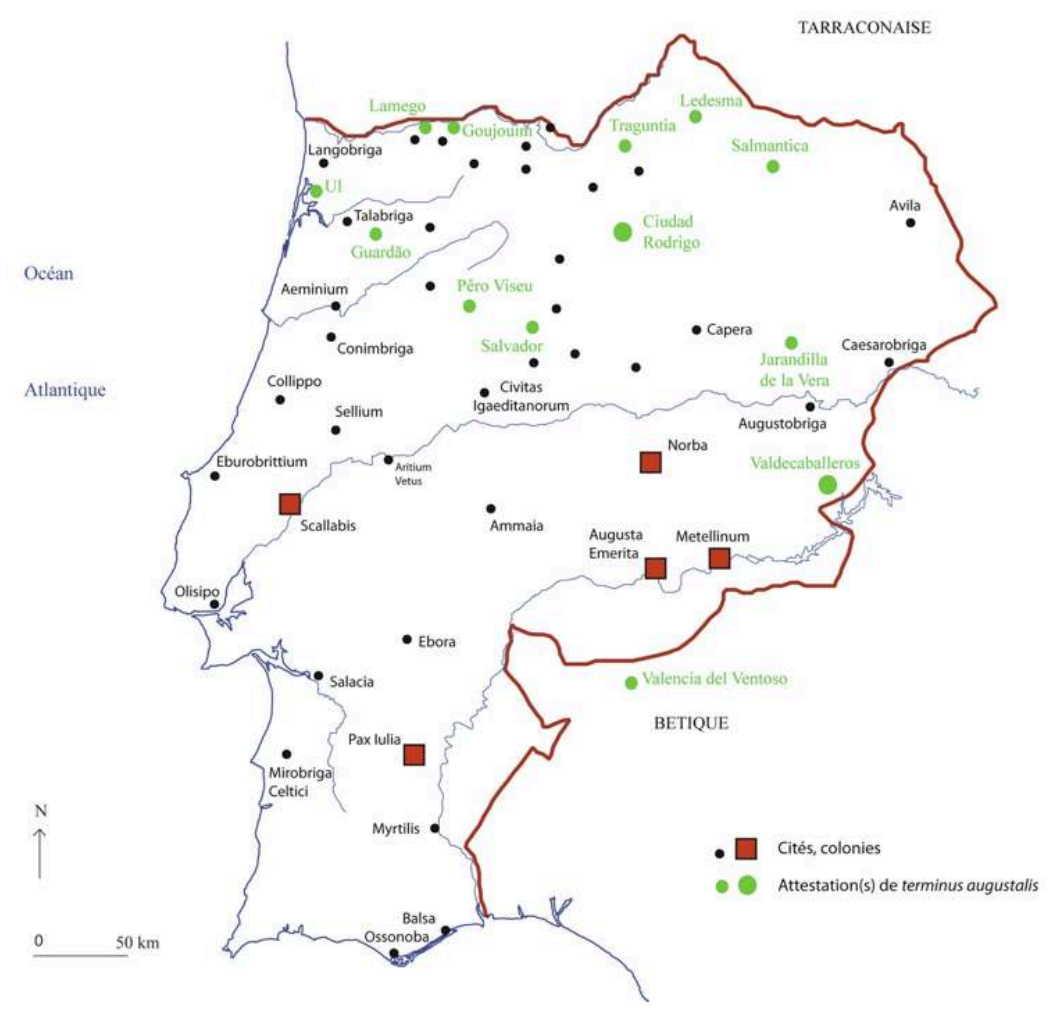

Dans ces régions qui n'ont jusque-là pas vraiment connu d'occupation romaine, on observe une véritable volonté de donner une assise territoriale, de fixer des peuples qui, pour beaucoup, ont vécu de la transhumance ${ }^{43}$. Ces pierres se font ainsi les témoins de la mise en place de nombreuses ciuitates à cette date. L'intervention d'Auguste est à ce titre exemplaire et revêt, dans ces lointaines contrées de l'Empire, un caractère politique. Au contraire, aucun témoignage d'opération de ce genre n'est connu au sud du Tage : cela doit probablement être mis en relation avec le substrat ethnique et culturel organisé autour de cités-États et l'ancienneté de la présence romaine. En effet, tandis que le sud lusitanien est intégré à la province de l'Vlterior ${ }^{44}$ et au modèle de la ciuitas dès l'époque républicaine, au contraire, la conquête est progressive, plus tardive, 
dans le nord, dans des contrées également plus montagneuses : là, il est plus difficile, encore aujourd'hui, de localiser les chefs-lieux de cités et le modèle urbanistique correspond d'ailleurs moins aussi aux canons romains ${ }^{45}$. Il n'est donc pas étonnant que, dans ces régions plus septentrionales et à cette époque, les ethniques soient utilisés pour faire référence à la communauté.

C'est précisément le cas de l'inscription romaine la plus ancienne de Lusitanie qui a été retrouvée dans la ciuitas Igaeditanorum (tableau 3$)^{46}$. Faute de sources attestant l'existence d'un oppidum préromain ${ }^{47}$, l'idée d'une fondation ex nihilo de la ciuitas vers 35 av. J.-C. est généralement acceptée. La date consulaire de 16 av. J.-C. justifie un certain archaïsme du texte, visible dans l'orthographe du nom Igaiditanis ou encore l'anthroponymie qui indique certains traits indigènes. La mention des magistrats préfigure les quattuouiri chargés de l'administration municipale romaine et confirme le caractère officiel de l'acte évergétique : il s'agit du don d'un horologium par un individu - dont nous ignorons tout par ailleurs -, mais dont l'inscription dans la tribu Papiria indique bien qu'il n'est pas originaire de la ciuitas Igaeditanorum. S'agirait-il de l'un des tout premiers colons d'Augusta Emerita qui relève de cette tribu? Quelles que soient les motivations d'un tel acte, il est évident que l'introduction d'un cadran solaire dans la vie publique de la communauté dès cette époque marque l'instauration de certaines pratiques sociales romaines: la communauté serait dorénavant régulée par la même heure que celle de la capitale de province. Par ailleurs, l'évergésie s'adresse ici à une collectivité d'habitants: les Igaeditani, nom qui reprend l'ethnique des populations locales et laisse entrevoir une certaine continuité entre le populus et la ciuitas qui réorganise le territoire. L'usage des mots dit beaucoup des conceptions et des représentations : ces régions septentrionales, au tout début de l'Empire, reflètent ce moment transitoire où l'ethnique prévaut encore pour désigner la communauté d'individus, comme un héritage du populus qui occupait ces terres avant l'arrivée des Romains.

10 Le glissement sémantique dans les inscriptions reflète l'évolution de la pensée et de l'organisation politique. La dédicace d'un citoyen romain «au Genius de l'oppidum constitué » est tout à fait révélatrice de la mise en place des nouveaux cadres romains à Ammai $^{48}$. Sans doute faut-il comprendre qu'il est question d'un vœu au numen qui protégeait le lieu avant la présence romaine, et qui est par la suite devenu la divinité tutélaire de la ciuitas. Si nous en ignorons la date exacte, cette dédicace n'est pourtant pas sans rappeler le texte de Pline et l'usage du terme oppidum. À l'époque augustéenne, la capitale de ciuitas peut, dans certains cas, correspondre à un oppidum lorsque celle-ci est installée sur le site d'origine: le noyau de peuplement est réutilisé pour les fonctions d'administration locale. Mais il n'en est pas toujours ainsi et ce n'est que par analogie que le mot est ensuite utilisé pour désigner le centre urbain. Ainsi, les multiples peuples sont intégrés sous la domination d'un lieu central, au sein d'une organisation politique hiérarchisée à une plus vaste échelle. L'expression «oppidum constitué » est donc révélatrice de la nouvelle réalité dont le choix de vocabulaire nous fait vivre cette entrée dans le cadre romain. L'adjectif constitutus doit être compris au sens "d'établi, d'organisé », formulation qui renvoie directement à la pensée cicéronienne de ciuitas constituta ${ }^{49}$. Ainsi, dans la cité cicéronienne peuvent entrer, en tant que citoyens à part entière, tous ceux qui acceptent de se soumettre aux droits et devoirs civiques, quelle que soit l'origine ethnique, la langue, ou l'histoire de l'individu 
ou de la communauté. C'est la raison pour laquelle la ciuitas est considérée comme le vecteur privilégié de la citoyenneté.

11 Sans aucun doute, la ciuitas est au cœur du système romain : c'est la cellule de base de l'administration d'un empire si étendu. Dès lors, l'usage progressif du terme ciuitas dans l'épigraphie traduit l'intégration politique au sein du cadre politique romain en même temps qu'il signifie aussi pour la communauté une promotion, une ascension dans la conception juridique urbaine qu'elle peut, en certaines occasions, afficher avec fierté. Si nous reprenons l'exemple précédent des Igaeditani dont il était question en 16 av. J.-C., nous pouvons nous rendre compte que seulement quelques années plus tard, dans un hommage officiel à Caius César daté de 1 à 4 apr. J.-C., la communauté se désigne elle-même - et ce point est fondamental - comme la ciuitas Igaeditanorum, c'est-à-dire la cité des Igaeditains ${ }^{50}$. À ce titre d'ailleurs, elle dispose de son propre personnel municipal, dont témoigne une seconde inscription qui signale un affranchi public ${ }^{51}$. Autrement dit, en quelques années, au moment clé de la réorganisation politique de toute la province, s'est produit un basculement de la désignation du seul ethnique à la mention de la ciuitas. Le nom même donné à la cité, forgé à partir de l'ethnique, montre que cette dernière est une réalité reconnue par les Romains. Le même schéma se retrouve dans les dédicaces à Jupiter Optimus Maximus, sous la protection duquel se placent deux autres cités du Nord lusitanien, la ciuitas Baniensis ${ }^{52}$ et la ciuitas Cobelcorum $^{53}$. Comme précédemment, la composition du nom, qui combine statut civique et ethnique, reflète à la fois le degré d'insertion dans le cadre municipal romain tout comme elle révèle une certaine identité du populus. Par conséquent, l'utilisation du pluriel traduit une réalité qui est celle de la communauté qui habite celle-ci, avec des réminiscences des liens de sang et de parenté héritées de la période précédente. De multiples peuples sont donc intégrés sous la domination d'un lieu central, au sein d'une organisation politique hiérarchisée. Par conséquent, puisque la constitution de la ciuitas implique la concession d'un statut privilégié, c'est aussi une promotion dans la hiérarchie politico-juridique pour l'oppidum choisi comme caput ciuitatis, et qui se voit directement confier par le pouvoir des fonctions politiques, économiques et religieuses. «D'un tissu lâche et capricieux de communautés préromaines, la République et surtout l'Empire ont fait un tissu ordonné, hiérarchisé. En offrant un modèle juridique, Rome a su donner à des ethnies si diverses, aux langues si mêlées une manière d'identité ${ }^{54} »$ : celle de la ciuitas.

12 Toutefois, à l'image du texte de Pline, d'autres inscriptions ne font pas mention de l'ethnique et insistent sur la ciuitas elle-même, comme Ossonoba et Ammaia: dédicace officielle, le texte exprime à chaque fois le cadre légal de l'époque. La première ${ }^{55}$ est un hommage de la ciuitas d'Ossonoba, sur la côte de l'Algarve, à son patron, également flamine provincial. Datée du $\mathrm{I}^{\mathrm{er}}$ siècle, comme l'indique l'orthographe de Lysitania, cette datation haute ne doit pas surprendre: l'influence culturelle romaine sur la partie méridionale de la province y est plus ancienne qu'ailleurs. L'emploi du terme ciuitas et le patronage civique municipal ${ }^{56}$ montrent à quel point la communauté a pleinement adopté les usages et le modèle civique romain. Dans le cas d'Ammaia ${ }^{57}$, à proximité de la vallée du Tage, il s'agit d'un vote annuel sur bloc de marbre en honneur à l'empereur Claude, en $44-45$, en présence du gouverneur de province. Même si l'onomastique montre encore à cette date que les magistrats de la cité sont des pérégrins, autant dire que c'est un acte de fidélité par excellence au pouvoir impérial. Ces deux inscriptions partagent donc plusieurs points communs. Tout d'abord le fait que ce soit la cité ellemême qui soit la dédicante de ces hommages; le caractère officiel de ces actes qui, de 
ce fait, se font dans le cadre légal qui est celui de l'autorité romaine : il est donc normal que le terme ciuitas soit employé ; enfin, l'appartenance de ces deux cités à la même aire celtico-turdule que nous avons déjà évoquée ne peut être négligée : ce sont ces mêmes communautés que Pline désignait comme oppida, sans mention d'un génitif pluriel. Un hommage impérial dédié à Hadrien par la ciuitas Aravorum en 117-118 - et donc bien plus tardif que les précédents - abonde aussi en ce sens ${ }^{58}$. Le texte reprend tous les codes des hommages impériaux, et pourtant, au II $^{\mathrm{e}}$ siècle, la cité se désigne encore comme la ciuitas Aravorum. Ainsi, si ces trois exemples montrent comment, progressivement, non seulement les communautés sont entrées dans les cadres romains, mais ont aussi pleinement adopté le fonctionnement municipal, il n'en reste pas moins vrai que des disparités, étroitement liées à l'histoire locale et au contraste nord/sud, persistent et doivent nuancer une vision trop simplificatrice.

13 En parallèle, l'usage épigraphique reflète également la hiérarchie des statuts juridiques, sources de prestige pour la communauté. À ce titre, par exemple, jamais une cité ne se dit pérégrine. Au contraire, lorsque le statut juridique est mentionné59, c'est généralement une manière d'afficher l'importance de la communauté, voire de dire sa promotion à un statut supérieur. C'est mettre ainsi en exergue l'autorité de la ciuitas et son statut. Le vocabulaire choisi pour qualifier la ciuitas reflète pleinement l'intégration aux cadres juridiques qui sont désormais ceux de la province. Un soin particulier est accordé à la mention de ces statuts lorsqu'il est question de la carrière de certains notables bien connus. C'est le cas par exemple à Augusta Emerita, qui est à la fois colonie et surtout capitale de province ${ }^{60}$. Plus le nombre de charges est important et le statut de la cité élevé, plus le prestige de la cité rejaillit sur l'individu. Ce pouvoir est démultiplié si les fonctions sont exercées dans des ciuitates distinctes. Le cas d'Ebora est en ce sens éclairant : le fait qu'une flaminique soit à la fois flaminica de la cité et de province est bien évidemment aussi source de prestige pour la communauté elle-même ${ }^{61}$. Il en est de même lorsque d'autres prêtresses ou augustales cumulent leurs fonctions dans de multiples cités de rangs distincts, municipes et colonies, et surtout de statut supérieur à la leur ${ }^{62}$. Le fait que l'individu exerce les mêmes fonctions dans une cité de rang supérieur lui donne à la fois davantage de pouvoir, de prestige, mais fait rejaillir cette promotion sur la ciuitas elle-même. Les mots affichés sont soigneusement choisis, pensés et disent beaucoup de ceux qui les ont fait graver. S'identifier, c'est s'affirmer dans le paysage urbain mais aussi promouvoir son importance. Ainsi, se nommer, c'est avant tout se dire.

14 L'analyse du lexique montre une réelle évolution au cours de l'époque impériale : une nouvelle étape est franchie avec l'usage d'un vocabulaire épigraphique plus spécifique, directement inspiré de Rome, qui exprime une certaine uniformisation des provinces. Dès lors, la Lusitanie ne fait plus figure de territoire lointain, périphérique de l'Empire. À partir du II ${ }^{\mathrm{e}}$ siècle, en effet, l'emploi de l'adjectif splendidissimus/a pour qualifier les «choses municipales » devient assez classique. Il désigne l'ordo ou encore directement la ciuitas. À l'heure actuelle, nous comptons quatre attestations de ce vocable en Lusitanie : une pour désigner la ciuitas, dans une inscription évergétique qui témoigne de la restauration des portes de Bobadela par la flaminique Iulia Modesta ${ }^{63}$; puis trois pour qualifier l'ordo. La première est une dédicace du II $^{e}$ siècle au dieu Esculape par l'un des médecins de la colonie de Pax Iulia, C. Attius Ianuarius ${ }^{64}$; la deuxième est un hommage public rendu à un gouverneur sous Septime Sévère $(146-211)^{65}$. L'emploi de l'adjectif pourrait s'expliquer à la fois par le contexte, la chronologie avancée, mais 
surtout le fait qu'il s'agisse d'Augusta Emerita, la capitale de province; enfin, la troisième attestation ${ }^{66}$ est, elle, plus surprenante : retrouvée dans la modeste cité de Capera, il s'agit d'une petite base de statue en or et argent en hommage à Iulia Augusta, mère des Castors. Datée d'environ 193-211, peut-être faut-il voir dans cet autre acte de loyalisme un certain mimétisme de la capitale de province et la volonté de mettre en exergue la richesse de la ciuitas qui est aussi un important centre minier ${ }^{67}$. Deux remarques émergent de ce rapide état des lieux. Tout d'abord concernant la chronologie. Si les inscriptions ne peuvent être datées plus précisément, elles semblent toutes être postérieures au $\mathrm{II}^{\mathrm{e}}$ siècle, date à laquelle émerge l'usage du terme splendidissimus/a dans l'épigraphie civique. Autrement dit, la Lusitanie suit le modèle impulsé par Rome, employant d'ailleurs ce vocabulaire avec un certain temps de décalage qui traduit le mouvement de diffusion. Expression des organes représentatifs de la cité, le lexique employé est celui du cadre civique officiel. Enfin, il est à noter que l'ordo reste toujours l'organe représentatif des habitants (ordo Emeritensium, ordo Caperensium), c'est-à-dire celui de la communauté qui se regroupe au sein de la ciuitas.

En parallèle, apparait le terme de res publica ${ }^{68}$ qui est désormais utilisé pour désigner la cité à proprement parler: il remplace alors l'usage de colonia ou municipium ${ }^{69}$. Ce nouveau lexique, connu par les lois municipales ${ }^{70}$, est illustré par le cas lusitanien (tableau 2).

Tableau 2. - Attestations du terme res publica dans l'épigraphie lusitanienne.

\begin{tabular}{|c|c|c|c|}
\hline $\mathbf{N}^{\circ}$ & Références & Extrait du texte & Commentaire \\
\hline 16 & $\begin{array}{l}\text { CIL, II, } 53(\text { ILS, } 6897 ; \text { ILER, } \\
1559)=I R C P, 239 \text { Pax Iulia }\end{array}$ & $o b /$ rem p(ublicam) bene / administraltam & $\begin{array}{l}\text { Grand bloc de marbre honorant un notable municipal } \\
\text { pour le zèle dont il a fait preuve dans sa gestion } \\
\text { municipale pendant son duumvirat, et pour ses actes } \\
\text { d'évergétisme. } I^{\text {er }} \text { siècle. }\end{array}$ \\
\hline 17 & HEp $, 11,703$ Sellium & $R(e s) p($ ublica $)$ S(eiliensis). & $\begin{array}{l}\text { Graffito avant cuisson sur une tegula fragmentée du } \\
\text { Ier siècle. Éventuellement liée à la construction et à la } \\
\text { réparation d'édifices publics, surtout lors de la } \\
\text { rénovation urbanistique impulsée par la promotion } \\
\text { flavienne dont bénéficie la cité. }\end{array}$ \\
\hline 18 & $\begin{array}{l}\text { CIL, II, } 1(\text { (ILS, } 534 ; \text { RAP, } \\
516)=I R C P, 3(H E p, 14,443) \\
\text { Ossonoba }\end{array}$ & $\begin{array}{l}\boldsymbol{r}(\text { (es) } \boldsymbol{p} \text { (ublica) Ossonob(ensis), / ex } \\
\text { decreto / ordin (is) / d(evota) n(umini) } \\
\text { m(aiestatique) eius / d(edit) d(edicavitque). }\end{array}$ & $\begin{array}{l}\text { Hommage de la cité à Valérien daté de la fin } 254-255 \text {. } \\
\text { En période de crise et d'invasions, Ossonoba affirme sa } \\
\text { loyauté envers Rome et démontre le bon fonctionnement } \\
\text { de ses institutions. }\end{array}$ \\
\hline 19 & $\begin{array}{l}A E, 1897,49=1900,31 \\
=I R C P, 4 \text { Ossonoba }\end{array}$ & $\begin{array}{l}\boldsymbol{r}(\text { es) } \boldsymbol{p} \text { (ublica) Ossonob(ensis), / ex } \\
\text { decreto / ordin(is) / d(evota) n(umini) } \\
\text { m(aiestatique) eius / d(edit) d(edicavitque). }\end{array}$ & $\begin{array}{l}\text { Idem que la précédente, mais cette fois-ci en hommage } \\
\text { de la cité à l'empereur Aurélien en } 274 \text {. }\end{array}$ \\
\hline 20 & $\begin{array}{l}\text { CIL, II, } 484 \text { (ILS, } 1372 ; \text { ILER, } \\
\text { 1529) Augusta Emerita }\end{array}$ & curatori rei pu/blicae Emerit(ae), & $\begin{array}{l}\text { Base de statue en hommage à un procurateur de la } \\
\text { province au III' siècle et curateur d'Augusta Emerita. }\end{array}$ \\
\hline 21 & IRCP, 75 Balsa & $\begin{array}{l}\text { ex decreto decuri]onum, } r(e s) \text { p(ublica }) \\
\text { Bals[ensium --]. }\end{array}$ & $\begin{array}{l}\text { Vu le caractère monumental des lettres sur marbre, il est } \\
\text { fort probable que l'inscription provienne de l'un des } \\
\text { monuments majeurs de la cité. Datation inconnue. }\end{array}$ \\
\hline 22 & CIL, II, 353 Eburobrittum & Callaecus / r(eipublicae) s(uae) l(ibertus) & $\begin{array}{l}\text { Inscription funéraire dont le dédicataire est un affranchi } \\
\text { public de la cité d'Eburobrittum. Datation inconnue. }\end{array}$ \\
\hline 23 & $H E p, 5,1032$ Ansião & $\begin{array}{l}\text { Ve(ctigale) } r(\text { (ei) p(ublicae) m(unicipii) } \\
\text { vicini. }\end{array}$ & $\begin{array}{l}\text { Cette inscription témoigne du souci des entités locales de } \\
\text { définir les territoires tributaires. Elle confirme ainsi que } \\
\text { la limite entre Sellium et Conimbriga passe par les } \\
\text { sommets de collines orientées est/ouest au sud de } \\
\text { Junqueira. Datation inconnue. }\end{array}$ \\
\hline
\end{tabular}

Sans revenir sur la complexité et la polysémie d'un concept aussi central dans le langage politique romain que la res publica ${ }^{71}$, et que nous ne saurions ici résoudre, quelques remarques peuvent néanmoins émerger de nos sources épigraphiques. À l'image de ses voisines africaine et bétique, en Lusitanie le terme res publica est employé indépendamment du statut juridique des cités $^{72}$. De façon assez classique également ${ }^{73}$, le concept est ici employé soit pour justifier l'octroi d'honneurs à un personnage en récompense de ses bienfaits ${ }^{74}$, soit pour désigner ce qui appartient à la 
communauté, c'est-à-dire les finances publiques ${ }^{75}$. Toutefois, le corpus ne permet pas d'éclairer davantage la connotation financière qui fait parfois débat. En effet, dans la mesure où dans les deux attestations où il est question de la gestion municipale et du vectigal $^{76}$ le terme res publica apparaît seul, sans mention de la communauté, ne pourrions-nous pas concevoir que le concept fait ici référence à la caisse publique ? Dans la majorité des autres cas, le terme est suivi du nom des habitants, ce qui semble distinguer la collectivité des citoyens : il s'agit généralement d'un hommage officiel à l'égard du pouvoir impérial pour lequel la res publica agit en tant que dédicante. Emmanuel Lyasse conclut : «[La res publica] est un terme de vocabulaire politique latin, que les Romains appliquent à toutes les formes de communautés politiques. On n'est ni plus romanisé, ni plus autonome, quand on qualifie sa communauté de res publica $"{ }^{77}$, l'assimilant davantage à un "phénomène de mode ». Malheureusement, le corpus ne permet pas de dépasser ce constat : tous les statuts juridiques sont concernés ainsi que tous les niveaux économiques et sociaux; toutes les attestations démontrent un même respect des cadres juridiques qui la rapprochent de « la république par excellence, la respublica populi Romani ${ }^{78} »$. D'une certaine manière, cette auto-identification juridique à Rome semble flatter les cités ${ }^{79}$. L'expression res publica, aux III $^{\mathrm{e}} \mathrm{II}^{\mathrm{e}}$ siècles av. J.-C., met surtout l'accent sur ce qui est en commun, par opposition aux choses privées: le terme res désigne tout autant les aspects matériels que politiques et administratifs de la vie commune. Ainsi, Servius Sulpicius Festus ${ }^{80}$ définit le municipe comme ayant une respublica différente de celle du peuple romain (« separatim a populo romano»), dans la mesure où ses habitants avaient accès à la citoyenneté romaine sans pouvoir occuper des magistratures romaines. Il avait donc sa propre gestion administrative et gouvernementale : des biens communs, un trésor, des magistrats, etc. Par conséquent, dans la définition de la res publica n'entrent ni l'idée ethnique, ni l'idée territoriale: Festus suggère ainsi que d'autres communautés, quel que fût leur statut juridique, pouvaient «rempublicam habere ${ }^{81}$ ». D'après Claudia Moatti ${ }^{82}$, le concept de res publica a connu une évolution sémantique au dernier siècle avant notre ère qui l'a fait passer d'une acception traditionnelle patrimoniale et politique - une res publica est un espace où des "choses " sont en partage, un tout doté d'institutions particulières - à un déplacement vers la "souveraineté » du peuple que traduit bien la définition de Cicéron $^{83}$ : la res publica, c'est la res populi, la « chose du peuple». Au sens où il s'agit de la définition de la collectivité même, il y a donc adéquation entre la res publica et la ciuitas. Une fois de plus, c'est bien le vocabulaire officiel qui est repris sur le modèle de l'autorité romaine, certes signe de son intégration au modèle mais qui dit peu de son usage au quotidien au sein de la communauté.

En conclusion, nommer la ville n'a rien d'anodin. Nommer la ville, c'est faire exister la cité et affirmer son statut. C'est prendre la pleine mesure de son rôle politique. Considérée avant tout comme le sceau de la réorganisation administrative de l'Empire, la ciuitas incarne une dimension essentielle dans la mesure où elle sert à délimiter, à organiser les terres conquises. Elle s'affirme avant tout comme le signe tangible de l'occupation de l'espace et de son appropriation. Avec la réorganisation augustéenne, toutes les communautés soumises à Rome sont incluses dans cette nouvelle unité, réordonnées d'une manière qui se veut plus efficace qu'auparavant. C'est donc là un instrument de maîtrise du territoire fondamental. Malgré les limites de nos sources, trop souvent partielles ou parfois difficilement datables, le vocabulaire permet d'aborder tour à tour l'imbrication du populus, de l'oppidum et de la ciuitas, d'envisager également la hiérarchisation juridico-administrative urbaine de l'Empire et les 
conceptions politiques qui leur sont intimement liées, et, parfois, d'approcher la réalité de la pratique quotidienne. Ainsi, les sources épigraphiques résument-elles une construction longue de plus de deux siècles. L'analyse lexicale dit beaucoup des communautés en fonction du choix des termes employés dont l'évolution chronologique permet de suivre la progression de la pensée politique. Théorisation politique et usage épigraphique se rejoignent ici. Le glissement significatif et progressif du vocabulaire au gré de la période impériale, et jusqu'au concept de res publica, éclaire ce moment clé où les populations conquises entrent dans une phase de transition, avant d'intégrer pleinement les cadres juridiques romains et ses conceptions politiques. Les termes utilisés reflètent non seulement la place de la communauté dans le cadre romain, mais aussi son adhésion à celui-ci. Ainsi, identifier une communauté, se nommer, c'est se dire en tant que ciuitas, voire affirmer son statut et jouir du prestige qui lui est lié. Reste à déterminer si les sources archéologiques confirmeraient aussi l'évolution que nous laisse entrevoir le vocabulaire : réalité du fait urbain ou simple discours officiel?

Tableau 3. - Corpus des inscriptions lusitaniennes ${ }^{84}$.

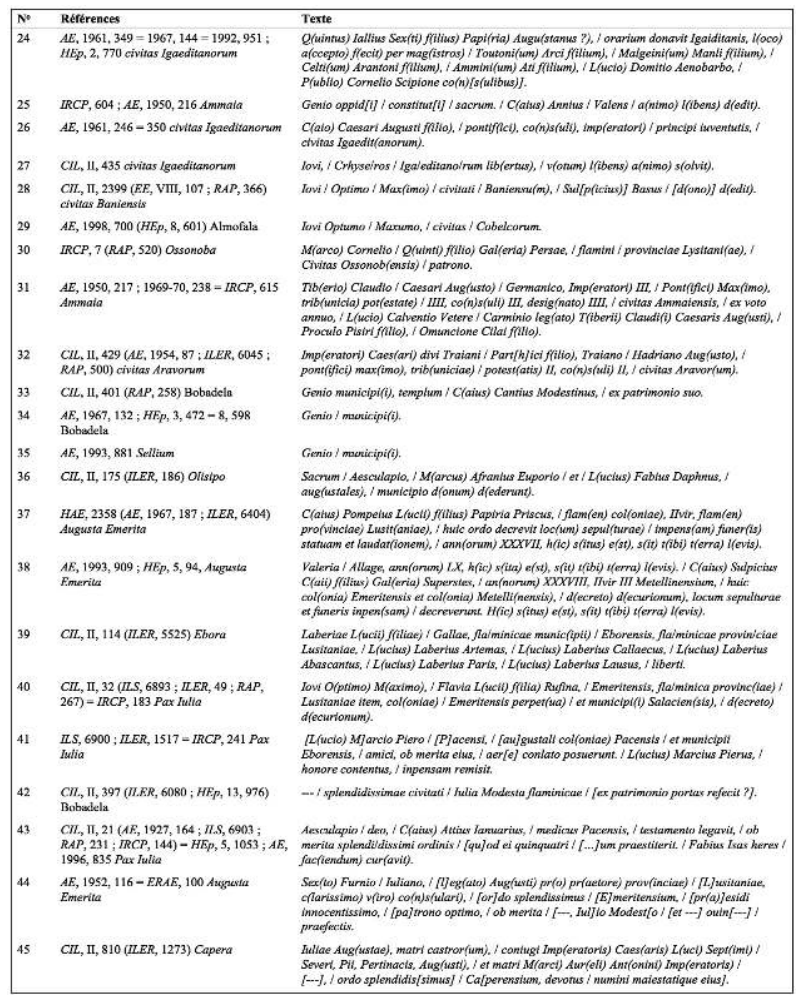

\section{BIBLIOGRAPHIE}

ALARCÃO Jorge de, Portugal romano, Lisbonne, Verbo, 1973. 
ALARCÃO Jorge de, O Domínio romano em Portugal, Lisbonne, Europa-América, 1988.

ALARCÃO Jorge de, «Identificação das cidades da Lusitânia portuguesa e dos seus territórios ", dans Les villes de Lusitanie romaine. Hiérarchies et territoires (Actes de la table ronde internationale du CNRS, Talence, 8-9 décembre 1988), Paris, CNRS, 1990.

ALARCÃO Jorge de, "On the civitates mentionned in the Inscription of the Bridge of Alcântara ", Journal of Iberian Archaelogy, 0, 1998, p. 143-157.

ALARCÃo Jorge de, « As cidades de Lusitânia: imagens de um processo cultural », dans T. Nogales Basarrate (éd.), Augusta Emerita. Territorios, Espacios, Imágenes y Gentes en Lusitania Romana, Mérida, MNAR, 2004.

ARIÑO Enrique, « La Hispania Citerior occidental y la Lusitania septentrional entre Augusto y los Flavios: el ager per extremitatem mensura comprehensus ", dans L'Aquitaine et l'Hispanie septentrionale à l'époque julio-claudienne. Organisation et exploitation des espaces provinciaux (Actes du IV colloque Aquitania, Saintes, 11-13 septembre 2003), Bordeaux, Fédération Aquitania, coll. « Suppléments Aquitania, 13 », 2005.

ARIÑO Enrique \& PAULE RUBIO Angel, « Una delimitación territorial de época de Vespasiano: dos inscripciones rupestres en el norte de la provincia de Cáceres (España) », Aquitania, 18, 2001, p. 411-419.

BONNEVILLE Jean-Noël, ÉTIENNE Robert, ROUILLARD Pierre, SILLIÈRES Pierre \& TRANOY Alain, "Les villes romaines de la péninsule Ibérique », dans Les villes dans le monde ibérique (Actes du colloque de Talence, 27-28 novembre 1980), Paris, CNRS, 1982.

CARVALHO Pedro, Cova da Beira. Ocupação e exploração do territorio na época romana, Fundão/ Coimbra, Câmara municipal de Fundão, Instituto de Arqueologia, 2007.

CARVALHO Pedro, «O forum dos Igaeditani e os primeiros tempos da civitas Igaeditanorum (Idanha-a-Velha, Portugal) », AEspA, 82, 2009, p. 115-131.

CARVALHO Pedro, «O interior norte de Lusitana romana. Resistencias, mudanzas e rupturas nos primeiros tempos do Imperio », dans I. Sastre \& A. Beltrán (éd.), El Bronce de El Picón (Pino del Oro). Procesos de cambio en el occidente de Hispania, Valladolid, JCYL, 2010.

CASTILLO PASCUAL María J., Espacio en orden: el modelo gromático-romano de ordenación del territorio, Logroño, Universidad de la Rioja, 1996.

CHOUQUER Gérard \& FAVORY François, Les arpenteurs romains. Théorie et pratique, Paris, Errance, 1992.

CORBIER Mireille, « Les mots de la ville et de la cité dans l'Empire romain », Topoi, Suppl. 12, 2013, p. 517-542.

CORTÉS Carolina, « Epigrafía y territorio en la Hispania romana: los termini públicos », Anas, 15-16, 2002-2003, p. 107-126.

CORTÉS Carolina, Epigrafía en los confines de las ciudades romanas: los termini publici en Hispania, Mauretania y Numidia, Rome, L'“Erma” di Bretschneider, 2013.

DARDAINE Sylvie, « Une image des cités de Bétique aux II ${ }^{\mathrm{e}}$ et III ${ }^{\mathrm{e}}$ siècles après J.-C. : l'emploi du terme respublica dans les inscriptions de la province ", dans Ciudad y comunidad cívica en Hispania, siglos II y III d. C. (Actes du colloque organisé par la Casa Velazquez et par le Consejo Superior de Investigaciones Cientificas, Madrid, 25-27 janvier 1990), Madrid, Casa de Velázquez, 1993.

DILKE Oswald A. W., Les arpenteurs de la Rome antique, Sophia-Antipolis, APDCA, 1995. 
DOMERGUE Claude, Les mines de la péninsule Ibérique dans l'Antiquité romaine, Rome, École française de Rome, 1990.

DONDIN-PAYRE Monique \& RAEPSAET-CHARLIER Marie-Thérèse (éd.), Cités, municipes, colonies. Le processus de municipalisation en Gaule et en Germanie sous le Haut-Empire romain, Paris, Publications de la Sorbonne, 1999.

EDMONDSON Jonathan, « Les provinces hispaniques et l'impact du pouvoir romain : l'exemple de la Lusitanie (fin du $\mathrm{I}^{\mathrm{er}}$ siècle av. J.-C.-fin du II ${ }^{\mathrm{e}}$ siècle apr. J.-C.) ", dans F. Hurlet (éd.), Rome et l'Occident. Gouverner l'Empire (II siècle av. J.-C.-II ${ }^{e}$ siècle apr. J.-C.), Rennes, Presses universitaires de Rennes, 2009, p. 253-286.

EDMONDSON Jonathan, «A Tale of Two Colonies: Augusta Emerita (Mérida) and Metellinum (Medellín) in Roman Lusitania », dans R. J. Sweetman (éd.), Roman Colonies in the First Century of Their Foundation, Oxford, Oxbow, 2011, p. 32-54.

ÉTIENNE Robert, « À propos du territoire d'Emerita Augusta (Mérida) », dans M. Clavel-Lévêque \& R. Plana (éd.), Cité et territoire, Paris, Presses universitaires de Franche-Comté, 1995, p. 27-32.

FICHTL Stephan, Les peuples gaulois. III ${ }^{e}-I^{\text {er }}$ siècles av. J.-C., Paris, Errance, 2004.

FUSTEL DE COULANGES Numa Denis, La Cité antique : étude sur le culte, le droit, les institutions de la Grèce et de Rome, Paris, Hachette, 1881.

GASCOU Jacques, «L'emploi du terme respublica dans l'épigraphie latine d'Afrique », MEFRA, 91, 1979 (1), p. 383-398.

GÓMEZ-PANTOJA Joaquín, " Un nuevo terminus Augustalis en Lusitania », dans A. Sartori \&

A. Valvo (éd.), Identità e autonomie nel mondo romano occidentale, Faenza, Grafico Lega, 2011, p. 291-317.

GONZÁLEZ Julián, « The Lex Irnitana: A New Copy of the Flavian Municipal Law », JRS, 76, 1986, p. 147-243.

GORGES Jean-Gérard \& RODRÍGUEZ MARTÍN Francisco G., « Los territorios antiguos de Mérida. Un estudio del territorium emeritense y de sus áreas de influencia », dans T. Nogales Basarrate (éd.), Augusta Emerita. Territorios, Espacios, Imágines y Gentes en Lusitania Romana, Mérida, MNAR, 2004, p. 93-128.

GORGES Jean-Gérard \& NOGALES BASARRATE Trinidad (éd.), Naissance de la Lusitanie romaine ( $I^{e r} s$. av. J.-C.- $I^{\text {er }}$ s. apr. J.-C.) / Origen de la Lusitania romana (siglos I a. C.-I d. C.) (Actes de la VII ${ }^{\mathrm{e}}$ Table ronde internationale sur la Lusitanie romaine, Toulouse, 8-9 novembre 2007), Toulouse/Mérida, MNARMuseo Nacional de Arte Romano, 2010.

GUERRA Amílcar, « A propósito dos conceitos de "Lusitano" e "Lusitânia” ", Palaeohispanica, 10, 2010, p. 81-98.

LAGUNA DURÁN Ana, « Dos nuevas inscripciones de época romana en la muralla de Salamanca », Habis, 49, 2018, p. 119-128.

LE ROUX Patrick, « Cités et territoires en Hispanie : l'épigraphie des limites », MCV, 31, 1994 (1), p. 37-51.

LE ROUX Patrick, «La ville romaine en Hispanie », dans L. Tavares Dias (éd.), Emergência e desenvolvimento das cidades romanas no norte da Península ibérica (Actas da mesa redonda), Porto, IPPAR, 1999a. 
LE ROUX Patrick, «Le territoire de la colonie auguste de Mérida. Réflexions pour un bilan », dans J.-G. Gorges \& F. G. Rodríguez Martín (éd.), Économie et territoire en Lusitanie romaine, Madrid, Casa de Velázquez, $1999 b$.

LE ROUX Patrick, «Peuples et cités de la péninsule Ibérique du $\mathrm{II}^{\mathrm{e}}$ s. a. C. au $\mathrm{II}^{\mathrm{e}}$ s. p. C. », Pallas, 80, 2009, p. 147-173.

LE ROUX Patrick, « Les colonies et l'institution de la province romaine de Lusitanie », dans J.-G. Gorges \& T. Nogales Basarrate (éd.), Naissance de la Lusitanie romaine ( $I^{e r}$ s. av. J.-C.- $I^{\text {er }}$ s. apr. J.-C.) / Origen de la Lusitania romana (siglos I a. C.-I d. C.) (Actes de la VII ${ }^{\mathrm{e}}$ Table ronde internationale sur la Lusitanie romaine, Toulouse, 8-9 novembre 2007), Toulouse/Mérida, MNAR-Museo Nacional de Arte Romano, 2010, p. 69-91.

LE ROUX Patrick, « Cités et territoire : la question des relations », dans L. Lamoine, C. Berrendonner \& M. Cébeillac-Gervasoni (éd.), Gérer les territoires, les patrimoines et les crises. Le quotidien municipal II, Clermont-Ferrand, Presses universitaires Blaise Pascal, 2012, p. 21-33.

LE ROUX Patrick, «Chapitre $\mathrm{X}$. Les villes de la péninsule Ibérique romaine : un siècle d'historiographie », dans P. Le Roux, Espagnes romaines. L'empire dans ses provinces, Rennes, Presses universitaires de Rennes, 2014, p. 189-209.

LE ROUX Patrick, «Les inscriptions et l'histoire provinciale de la Lusitanie : un bilan », dans T. Nogales Basarrate (éd.), Lusitania romana. Del pasado al presente de la investigación, Mérida, MNAR-Museo Nacional de Arte Romano, 2017, p. 23-45.

Les villes de Lusitanie romaine. Hiérarchies et territoires (Actes de la table ronde internationale du CNRS, Talence, 8-9 décembre 1988), Paris, CNRS, coll. « La Maison des pays ibériques, 42 », 1990.

LEVEAU Philippe, « La ville romaine et son espace territorial », dans La Ciutat en el món romà (Actas del XIVè Congrés international d'Arqueologia Clàssica), Tarragone, Institut d'Estudis Catalans, 1993, p. 273-284.

LEVEAU Philippe, «Territorium Urbis. Le territoire de la cité romaine et ses divisions : $d u$ vocabulaire aux réalités administratives », REA, 95, 1993b (3-4), p. 449-471.

LYASSE Emmanuel, «L'utilisation des termes res publica dans le quotidien institutionnel des cités. Vocabulaire politique romain et réalités locales ", dans C. Berrendonner, M. Cébeillac-Gervasoni \& L. Lamoine (éd.), Le quotidien municipal dans l'Occident romain (Actes du colloque des 19, 20 et 21 octobre 2007), Clermont-Ferrand, Presses universitaires Blaise Pascal, 2008, p. 187-202.

MANTAS Vasco, «As civitates: esboço da geografia política e económica do Algarve romano », dans M. F. Barrata \& R. Pareira (éd.), Noventa séculos entre terra e mar, Lisbonne, IPPAR, 1997, p. 283-309.

MAYER I OLIVÉ Marc, «El concepto de res publica en época romana. Dos aspectos para un análisis de la evolución de su significado: el De Republica de Cicerón y la presencia posterior del término en la epigrafía », dans L. M. Bernardo, L. Santa Bárbara \& L. Andrade (éd.), Representações da República, Vila Nova de Famelicão, Edições Húmus, 2013, p. 287-300.

MELCHOR GIL Enrique, El Patronato cívico en la Hispania romana, Séville, Universidad de Sevilla, 2018.

MOATTI Claudia, Archives et partage de la terre dans le monde romain (II ${ }^{e}$ siècle avant- $I^{e r}$ siècle après J.-C.), Rome, École française de Rome, 1993.

MOATTI Claudia, « Respublica et droit dans la Rome républicaine », MEFRA, 113, 2001 (2), p. 811-837. 
MOATTI Claudia, Res publica. Histoire romaine de la chose publique, Paris, Fayard, 2018.

NOGALES BASARRATE Trinidad (éd.), Lusitania romana. Del pasado al presente de la investigación, Mérida, MNAR-Museo Nacional de Arte Romano, 2017.

OREJAS Almudena \& SASTRE Inés, « Fiscalité et organisation du territoire dans le nord-ouest de la péninsule Ibérique : civitates, tribut et ager mensura comprehensus », DHA, 25, 1999 (1), p. 159-188.

RAEPSAET-CHARLIER Marie-Thérèse, « Chapitre IV. Les Gaules et les Germanies », dans C. Lepelley (éd.), Rome et l'intégration de l'Empire, 44 av. J.-C.-260 apr. J.-C, t. 2 : Approches régionales du Haut-Empire romain, Paris, PUF, 1998, p. 143-195.

RAMÍREZ SÁDABA José Louis, « Augusta Emerita. La aportación de la epigrafía desde su fundación hasta la conquista por los árabes ", dans T. Nogales Basarrate (éd.), Lusitania romana. Del pasado al presente de la investigación, Mérida, MNAR-Museo Nacional de Arte Romano, 2017, p. 91-103.

RODRÍGUEZ Pilar, « El significado de civitas en Cicerón », Veleia, 7, 1990, p. 233-241.

SALINAS DE FRÍAS Manuel, « Las ciudades des poder de la provincia de Lusitania. Capitales conventuales y ciudades principales », Revista de Historiografía, 25, 2016, p. 133-155.

SÁNCHEZ-PALENCIA Francisco J. \& CURRÁS Brais X., « Minería del oro y explotación del territorio de Lusitania: estudo de la investigación », dans T. Nogales Basarrate (éd.), Lusitania romana. Del pasado al presente de la investigación, Mérida, MNAR-Museo Nacional de Arte Romano, 2017, p. 393-415.

SÁNCHEZ-PALENCIA Francisco J. \& PÉREZ GARCÍA Luis C., « Minería romana de oro en las cuencas de los ríos Erges / Erjas y Bazágueda (Lusitania): la zona minera de Penamacor-Meimoa », dans Lusitanos e Romanos no Nordeste da Lusitânia (Actas das $2^{\text {as }}$ Jornadas de Património da Beira Interior), Guarda, centre Estudos Ibéricos, 2005, p. 267-307.

ZECCHINI Giuseppe (éd.), « Ricostruzioni di una repubblica », Studi storici, 47, 2006 (2), p. 317-404.

\section{NOTES}

1. Je tiens à remercier les organisateurs de la journée d'étude de leur invitation à prendre part à la réflexion et aux échanges sur ce thème en mai 2018 à l'université de Dijon (ARTEHIS-UMR 6298 et Archimède-UMR 7044).

2. "Cité et ville n'étaient pas des mots synonymes chez les Anciens. La cité était l'association religieuse et politique des familles et des tribus ; la ville était le lieu de réunion, le domicile et surtout le sanctuaire de cette association. » (Fustel de Coulanges, 1881, chap. IV)

3. La bibliographie sur le sujet est abondante. De ce fait, dans le cadre de ce travail, nous renverrons aux ouvrages de référence ainsi qu'à ceux qui sont d'une utilité première pour notre propos. Pour une réflexion théorique sur la diversité et la portée du vocabulaire employé pour parler de la cité, voir Corbier (2013).

4. Pour l'organisation d'un territoire en cité, voir Le Roux (1994, 37-39), Raepsaet-Charlier (1998) et Dondin-Payre \& Raepsaet-Charlier (1999).

5. Rodríguez (1990, 236-237) ; Le Roux (1999a, 236).

6. Le Roux (2014, 189-209).

7. Loin d'être exhaustive, nous ne pourrions manquer de citer les travaux de J. de Alarcão (p. ex. 1973, 1988, 1990, 1998 ou 2004) ou encore de V. Mantas (1997). De même, l'ouvrage Les villes de Lusitanie romaine (1990) est fondateur car, en proposant une nouvelle carte administrative de la Lusitanie, il ouvre la voie à toutes les problématiques spatiales et territoriales. 
8. La série d'ouvrages qui a fait suite au précédent fonde en grande partie les connaissances que nous avons de la Lusitanie. Pour notre propos, voir notamment Gorges \& Nogales Basarrate (2010) ou Nogales Basarrate (2017), auxquels s'ajoutent un certain nombre de monographies comme Domergue (1990). Concernant les villes comme centre de pouvoir, cf. de récents travaux tels Edmondson (2011) ou Salinas de Frías (2016).

9. Sur le contexte de réorganisation provinciale, voir Les villes de Lusitanie romaine (1990) ou plus récemment Edmondson (2009, 253-254) et Gorges \& Nogales Basarrate (2010).

10. Voir Le Roux (2009 et 2010) pour une synthèse de cette mise en ordre administrative de la péninsule Ibérique et le passage des ethnies aux cités.

11. Pline l'Ancien, Histoire naturelle, IV, 113, 116 et 117-118.

12. Le Roux (1999a, 236).

13. Guerra (2010) insiste sur l'ambiguïté de l'usage différencié des concepts de Lusitanie et Lusitanus en fonction du contexte et des époques.

14. Pline l'Ancien, Histoire naturelle, IV, 117.

15. Strabon, III, $3,3:$ : [Ces peuples] trop petits et trop obscurs ne valent pas la peine qu'on les nomme. » Il remarque aussi que les populations ne parlent pas toutes la même langue et utilisent des alphabets différents (III, 1, 6).

16. Carvalho $(2007,58)$.

17. Carvalho $(2007,55-56)$ préfère de ce fait parler « d'esprit lusitanien ».

18. Voir Fichtl (2004) pour une étude complète du concept ainsi que le travail présenté par S. Lefebvre dans ce même ouvrage et dont la notion d'oppidum est au cœur de la réflexion.

19. Pline l'Ancien, Histoire naturelle, IV, 116 et 117.

20. Ibid., IV, 118 .

21. Bonneville et al. (1982, 11-13). D'ailleurs, Strabon, III, 3, 5 témoigne de l'existence de poleis.

22. Dans sa description administrative de la Narbonnaise, Pline l'Ancien, Histoire naturelle, III, 32-37, utilise de la même manière le terme oppidum de façon indifférenciée pour désigner les oppida latina, d'autres qui n'ont pas le statut latin, mais aussi des « oppida uero ignobilia ».

23. Rodríguez (1990, 234 et 237). Pour Le Roux (1999a, 236-237), tout populus inclut l'idée d'une communauté politique organisée; l'oppidum se réfère, lui, à un centre politique, un chef-lieu préexistant qui n'est d'ailleurs pas forcément en hauteur, alors que la ciuitas met l'accent sur l'autonomie locale.

24. Moatti $(1993,3)$.

25. Sur ces questions, voir plus particulièrement Leveau (1993 et 1993b) et Le Roux (1994, 1999 et 2012) dont les travaux reposent à la fois sur une réflexion conceptuelle du vocabulaire et sur l'étude de l'épigraphie dite «des limites ». Pour reprendre Le Roux (1999a, 245 ; 2012, 22-24), le concept de finis, que l'on peut traduire par «frontière » ou «confins », est utilisé pour rendre l'idée d'un espace bien borné et délimité, juridiquement et religieusement protégé. La « limite » est à mettre en lien avec la notion de limes, c'est-à-dire la ligne de confins entre deux espaces, ceux qui appartiennent à l'Empire, et les autres, mais son origine étymologique désigne aussi le «sentier» ou «sillon» en agriculture qui renvoie précisément aux mythes fondateurs (Dilke, $1995,41)$. Pour la liste complète des termini augustales de péninsule Ibérique, voir Cortés (2013, 221-223) ; Ariño (2005) pour une lecture d'ensemble du corpus lusitanien.

26. Frontin, Deagrorum qualitate, I, 1-2 (éd. Thulin): «ager per extremitatem mensura comprehensus ». Pour l'ensemble du processus (recensement, bornage, répartition des terres, etc.), voir Chouquer \& Favory $(1992,37-38)$, Dilke $(1995,94-98)$ et plus particulièrement Moatti (1993, 9-30) et Cortés (2013).

27. Hygin le Gromatique, I, 26-28. Nous n'entrerons pas ici dans les larges débats dont ce territoire a fait l'objet afin d'en déterminer les limites exactes. Pour une synthèse historiographique, voir Le Roux (1999b, 263-264), Gorges \& Rodríguez Martín (2004, 95-98) et Ramírez Sádaba (2017). 
28. Orejas \& Sastre (1999).

29. Dilke (1976, 98-108); Castillo Pascual (1996, 53-57) ; Cortés (2002-2003, 109-110) ; Ariño $(2005,109)$.

30. Notre propos n'est pas ici de revenir sur les débats concernant les délimitations des ciuitates et les questions des termini : pour une synthèse, voir Cortés (2013). Par ailleurs, seules ont été prises en compte les inscriptions concernant le territoire civique : les prata ont donc été laissés de côté. Enfin, pour les deux inscriptions rupestres, nous nous permettons de renvoyer à Ariño \& Paule Rubio (2001) et Ariño (2005, 108-109).

31. Nous en conservons deux exemplaires : $n^{\circ} 14$ et $n^{\circ} 15$ du tableau 1 , tous deux concernant la colonie d'Augusta Emerita.

32. Chez les Romains, toute délimitation spatiale revêt un caractère sacré : la fête des Terminalia a notamment pour but de renouveler la force protectrice des termini et le respect des propriétés. Ainsi, renverser, déplacer une borne peut être compris comme un acte d'impiété. Cette dimension religieuse garantit le respect de la décision impériale.

33. Ariño $(2005,103)$.

34. Cortés (2002-2003, 112).

35. La seule exception est le cas de l'inscription $n^{\circ} 15$ qui ne fait mention que d'un seul territoire, celui de la colonie capitale de province, Augusta Emerita.

36. Ariño $(2005,96-97)$.

37. $\mathrm{N}^{\mathrm{os}} 2,3$ et 4 .

38. $\mathrm{N}^{\text {os }} 5,6,7,8,9$ et 10 .

39. $\mathrm{N}^{\mathrm{os}} 11$ et 12 .

40. $N^{\text {os }} 13$ et 14 .

41. Ariño $(2005,103-104)$ met d'ailleurs l'accent sur la spécificité lusitanienne par rapport aux autres provinces hispaniques.

42. $\mathrm{N}^{\text {os }} 5,6,7,8$ et 9.

43. Étienne (1995, 29) ; Ariño (2005, 102-103).

44. Pline l'Ancien, Histoire naturelle, III, 6.

45. Par exemple, malgré les fouilles menées, aucune ville n'a pu être identifiée pour Lancia Oppidana en raison de la modestie urbaine de ce centre : cf. Carvalho $(2007,353)$.

46. Cf. tableau 3 : Corpus des inscriptions auquel renvoient les numéros indiqués désormais en référence. Ici, $\mathrm{n}^{\circ} 24$ : orarium donauit Igaiditanis.

47. Confirmé par les fouilles du centre civique menées en 2007-2008 : cf. Carvalho (2009, 117-120 et 125$)$.

48. $\mathrm{N}^{\mathrm{o}} 25$ : Genio oppid $[i]$ / constitut $[i]$.

49. Voir Mayer i Olivé (2013) pour une synthèse éclairante sur l'œuvre de Cicéron.

50. $\mathrm{N}^{\circ} 26$ : ciuitas Igaedit(anorum).

51. $\mathrm{N}^{\mathrm{0}} 27$ : Iga/editano/rum lib(ertus).

52. $\mathrm{N}^{\mathrm{0}} 28$ : ciuitati / Baniensu $(\mathrm{m})$.

53. $\mathrm{N}^{\circ} 29$ : cilitas / Cobelcorum.

54. Bonneville et al. $(1982,22)$.

55. $\mathrm{N}^{\mathrm{o}} 30$ : Ciuitas Ossonob(ensis).

56. Melchor Gil (2018) pour une synthèse de la question.

57. $\mathrm{N}^{\circ} 31$ : ciuitas Ammaiensis. À noter, une erreur du lapicide dans le nombre de saluts impériaux et la puissance tribunicienne.

58. $\mathrm{N}^{\circ} 32$ : ciuitas Arauor(um).

59. $\mathrm{N}^{\circ} 33$ et $\mathrm{n}^{\circ} 34$ Bobadela, $\mathrm{n}^{\circ} 35$ Sellium: Genio municipi(i); et $\mathrm{n}^{\circ} 36$ Olisipo: aug(ustales), / municipio d(onum) d(ederunt).

60. $\mathrm{N}^{\circ} 37$ : flam(en) col(oniae), Iluir, flam(en) pro(uinciae) Lusit(aniae) et $\mathrm{n}^{\circ} 38$ : huic col(onia) Emeritensis et col(onia) Metelli(nensis)... 
61. $\mathrm{N}^{\circ} 39$ : fla/minicae munic(ipii) / Eborensis, fla/minicae prouin/ciae Lusitaniae.

62. $\mathrm{N}^{\circ} 40$ : fla/minica prouinc(iae) / Lusitaniae item, col(oniae) / Emeritensis perpet(ua) / et municipi(i) Salacien(sis) ou $n^{\circ} 41$ : [au]gustali col(oniae) Pacensis / et municipii Eborensis.

63. $\mathrm{N}^{\circ} 42$ : --- / splendidissimae ciuitati.

64. $\mathrm{N}^{\circ} 43$ : ob merita splendi/dissimi ordinis.

65. $\mathrm{N}^{\circ} 44$ : [or]do splendissimus / [E]meritensium.

66. $\mathrm{N}^{\mathrm{o}} 45$ : ordo splendidis[simus] / Ca[perensium ...].

67. Pas moins de trois mines d'or y sont connues: Río Ponsul, Monfortinho et Meimoa; cf. Domergue $(1990,208)$, plus récemment Sánchez-Palencia \& Pérez García (2005). Voir SánchezPalencia \& Currás (2017) pour un état de la question sur l'exploitation de l'or en Lusitanie.

68. Polybe, 6, 51, 6 ; Cicéron, De Republica.

69. Corbier $(2013,535)$ estime que c'est globalement le cas à partir de l'époque sévérienne.

70. Voir les lois municipales d'époque impériale comme la Lex Irnitana: cf. González (1986).

71. Voir Zecchini (2006) pour l'analyse historiographique tout au long du $\mathrm{xx}^{\mathrm{e}}$ siècle. Voir plus particulièrement l'incontournable ouvrage de Moatti (2018, 25 et suiv.).

72. Respectivement Gascou $(1979,395-396)$ pour l'Afrique et Dardaine $(1993,48)$ pour la Bétique. Confirmé par Lyasse $(2008,188)$.

73. Lyasse (2008, 189-193).

74. Par exemple, $\mathrm{n}^{\circ} 16$ Pax Iulia : ob bene administratam.

75. Dans ce cas, le seruus rei publicae $\left(\mathrm{n}^{\circ} 22\right)$ pourrait être considéré comme un bien matériel d'Eburobrittum.

76. Respectivement $\mathrm{n}^{\circ} 16$ Pax Iulia et $\mathrm{n}^{\circ} 23$ Ansião.

77. Lyasse $(2008,199-200)$.

78. Dardaine $(1993,55)$.

79. Moatti $\left(2018,3^{\mathrm{e}}\right.$ partie $)$.

80. Festus, s. v. Municipium, p. 155 L.

81. Festus, s. v. Praefecturae, p. 262 L.

82. Moatti (2018, 196-198). Voir aussi Mayer i Olivé (2013).

83. Cicéron, De Republica, I, 25, 39 : Est igitur respublica res populi; populus autem non omnis hominum coetus quoquo modo congregatus, sed coetus multitudinis iuris consensu et utilitatis communione sociatus... Comme le fait remarquer Moatti $(2001,824)$, ce qui rend cette définition originale dans la tradition latine, c'est le déplacement de l'accent de la res au populus et à la notion de societas.

84. Pour rappel, les inscriptions référençant les termini augustales sont classées dans le tableau 1 et celles faisant mention de la res publica sont réunies dans le tableau 2.

\section{RÉSUMÉS}

C'est dans un contexte de réorganisation des communautés des provinces conquises au HautEmpire qu'est mise en place la ciuitas. En ce sens, la source épigraphique révèle tout autant la manière d'y faire référence que la diversité lexicale - et la palette d'adjectifs - pour nommer et qualifier la ville au sens large. Aussi, nous tenterons de faire le point sur les aspects juridiques et théoriques de ces notions, avant de revenir sur leur usage dans la pratique quotidienne, reflet de l'évolution des conceptions politiques. 
In the territories conquered in the early Empire, the juridical hierarchy of civic communities appears in the context of the administrative prouinciae reorganization. In this respect, the epigraphical study reveals not only the way to identify it, but also the lexical diversity to name and consider the ciuitas. Therefore, this paper aims, first at evaluating the theoretical and juridical aspects of this concepts, and second at questioning its daily experience that express the evolution of political ideas.

\section{INDEX}

Mots-clés : ciuitas, populus, res publica, épigraphie, Lusitanie

Keywords : ciuitas, populus, res publica, epigraphy, Lusitania

\section{AUTEUR}

\section{SUSANA MARCOS}

CY Cergy Paris Université, CRESEM - EA 7397

marcos.susana@hotmail.fr 